\title{
A decisão clínica compartilhada diante dos riscos do rastreamento do câncer de próstata
}

\section{The shared clinical decision regarding the risks of prostate cancer screening \\ La decisión clínica compartida con respecto a los riesgos del tamizaje del cáncer de próstata}

\author{
Renata Oliveira Maciel dos Santos ${ }^{1 \oplus}$, Mirhelen Mendes de Abreu $^{2 \oplus}$, Elyne Montenegro Engstrom ${ }^{3 \oplus}$ \\ ${ }^{1}$ Tecnologista no Instituto Nacional do Câncer. \\ 2 Universidade Federal do Rio de Janeiro (UFRJ). \\ ${ }^{3}$ Escola Nacional de Saúde Pública (Ensp/Fiocruz).
}

\section{Resumo}

Introdução: Evidências atuais apontam para desiquilíbrio entre os benefícios e danos com a prática do rastreamento do câncer de próstata, podendo provocar consideráveis riscos à saúde dos homens. Tal afirmativa fundamentou a recomendação do Ministério da Saúde sobre não estimular a realização dessa prática e a necessidade de discutir todas as implicações desses exames quando o homem solicitar sua realização. Objetivo: Analisar a percepção e experiências de homens e médicos/as sobre a tomada de decisão compartilhada para o rastreamento do câncer de próstata no Brasil. Método: Foi realizado um estudo qualitativo e exploratório, a partir de um grupo focal com homens na maturidade da idade (média: 51 anos) e entrevistas semiestruturadas com médicos/as de unidades básicas de saúde, de diferentes estados do Brasil. Abordagem analítica qualitativa, cujos dados foram categorizados pela análise de conteúdo. Resultados: Identificou-se desconhecimento dos homens sobre os possíveis danos relacionados ao rastreamento desse câncer, além de barreiras de cunho pessoal, cultural e na organização do processo de trabalho, que demonstraram dificultar à comunicação clínica durante a consulta na atenção primária à saúde (APS). De outra parte, os homens referem desejo de maior participação nas escolhas sobre sua saúde, mencionando a internet como uma ferramenta frequentemente utilizada para diminuir a disparidade de conhecimento na relação clínica. Os achados também apresentam estratégias utilizadas pelos médicos/as, como recursos gráficos e ferramentas de comunicação, que ajudam a incluir diferentes perfis de homens na tomada de decisão. Conclusão: A investigação demonstrou que, embora haja avanços em relação ao debate de possíveis implicações danosas do rastreamento do câncer de próstata e exemplos de abordagens inclusivas, essa prática ainda não está consolidada no Brasil, revelando a necessidade de incentivar a participação do homem nas decisões que influenciam diretamente sua qualidade de vida e bem-estar. Dessa forma, há a necessidade de investir em estratégias que facilitem a comunicação médico/paciente e ampliem o debate sobre as possíveis implicações do rastreamento entre os profissionais inseridos na APS e na população.

Palavras-chave: Neoplasias da Próstata; Comunicação em Saúde; Tomada de Decisão Compartilhada; Prevenção Quaternária.

Como citar: Santos ROM, Abreu MM, Engstrom EM.Decisão clínica compartilhada. Rev Bras Med Fam Comunidade. 2021;16(43):2470. https://doi.org/10.5712/rbmfc16(43)2470

Autor correspondente: Renata Oliveira Maciel dos Santos. E-mail: renamsant@yahoo.com.br Fonte de financiamento: declaram não haver.

Parecer CEP:

2.286.124

Procedência: não encomendado. Recebido em: 18/04/2020. Aprovado em: 12/01/2021. Editor Associado: Bruno José Barcellos Fontanella. 


\begin{abstract}
Introduction: Current evidence shows an imbalance between benefits and harms with the prostate cancer screening, which can cause considerable risks to men's health. This statement supports the recommendation of the Brazilian Health Ministry that recommends not to stimulate this practice and large discussion about the implications of these exams when the man requests it. Purpose: Analyze the perception and experience of men and doctors about sharing the decision in screening for prostate cancer. Method: A qualitative and exploratory study was carried out, from a focus group with men of mature age (average: 51 years) and semi-structured interviews with doctors in basic health units, from different states in Brazil. The data were systematized, grouped, and categorized by content analysis. Results: Men were unaware of the possible damages related to the prostate cancer screening, and we also identified personal, cultural barriers and the organization of work's process, that showed to difficult clinical communication during consultation in primary health care. On the other hand, men report a desire for greater participation in the choices about their health, mentioning the internet as a tool used to reduce the knowledge gap in the clinical relationship. The findings also present strategies used by doctors, such as infographic and tools, which help to include different profiles of men in decision making. Conclusion: The investigation demonstrated that, although there are advances in relation to the debate about possible harmful implications of prostate cancer screening and examples of inclusive approaches, this practice is not consolidated in Brazil, revealing the need to disseminate actions that encourage the participation in the decisions that directly influence their quality of life and well-being. Thus, there is a need to invest in strategies that facilitate doctor/patient communication, in addition to expanding the debate on the implications of screening among professionals inserted in Primary Health care and in the population.
\end{abstract}

Keywords: Prostatic Neoplasms; Health Communication; Decision Making, Shared; Quaternary Prevention.

\title{
Resumen
}

Introducción: La evidencia actual apunta a un desequilibrio entre los beneficios y los daños con la práctica del tamizaje del cáncer de próstata, que puede causar riesgos considerables para la salud de los hombres. Esta declaración respalda la recomendación del Ministerio de Salud Brasileño de que esta práctica no debe ser alentada y debe ser discutidas todas las implicaciones de estos exámenes cuando el hombre lo solicite. Objetivo: Analizar la percepción y experiencia de hombres y médicos acerca de compartir la decisión en la detección del cáncer de próstata. Método: se realizó un estudio cualitativo y exploratorio, de un grupo focal con hombres en edad madura (promedio: 51 años) y entrevistas semiestructuradas con médicos de unidades básicas de salud en diferentes estados de Brasil. Enfoque analítico cualitativo, cuyos datos fueron categorizados mediante análisis de contenido. Resultados: Los hombres desconocían el posible daño relacionado con el tamizaje cáncer de próstata, además fueran identificado barreras de carácter personal, cultural y organizativo en el proceso laboral, que demostraron dificultar la comunicación clínica durante la consulta en la atención primaria de salud. Por otro lado, los hombres manifiestan un deseo de mayor participación en las decisiones sobre su salud, y mencionan internet como una herramienta utilizada para reducir la brecha de conocimiento en la relación clínica. Los médicos también presentan estrategias utilizadas, como recursos gráficos y herramientas, que ayudan a incluir diferentes perfiles de hombres en la toma de decisiones. Conclusión: La investigación demostró que, si bien existen avances con relación al debate sobre posibles implicaciones nocivas del tamizaje del cáncer de próstata y ejemplos de abordajes inclusivos, esta práctica aún no está consolidada en Brasil, revelando la necesidad de difundir acciones que incentiven la participación de los hombres en las decisiones que influyen directamente en su calidad de vida y bienestar. Por lo tanto, es necesario invertir en estrategias que faciliten la comunicación médica, además de ampliar el debate sobre las implicaciones del tamizaje entre los profesionales insertados en la atención primaria de salud y en la población.

Palabras clave: Neoplasias de la Próstata; Comunicación en Salud; Toma de Decisiones Conjunta; Prevención Cuaternaria.

\section{INTRODUÇÃO}

A tomada de decisão compartilhada é entendida como o envolvimento dos indivíduos na decisão clínica, que preconiza o contexto de vida e as expectativas como principal orientadora do cuidado. ${ }^{1}$ Tem por objetivo garantir que os valores e preferências individuais sejam considerados no momento da decisão, respeitando o princípio da autonomia para escolhas que afetam a qualidade de vida e bem-estar. ${ }^{2}$

O referencial da decisão compartilhada dialoga com o método clínico centrado na pessoa, abordagem utilizada na formação e nas práticas da Atenção Primária à Saúde (APS), cujos componentes exploram a saúde, a doença e a experiência individual; o entendimento da pessoa como um todo; um plano de cuidado compartilhado e a construção contínua da relação médico/paciente. O método contrapõe a hegemonia do modelo biomédico, valoriza a coparticipação dos indivíduos no cuidado e preconiza relações mais simétricas nas decisões clínicas. ${ }^{3}$ 
Normativas brasileiras, como a Política de Atenção Básica, da Promoção de Saúde e da Humanização ${ }^{4-6}$ têm reafirmado ao longo das últimas duas décadas, modelos de atenção centrados na autonomia e participação do indivíduo no cuidado integral. Isto é particularmente necessário em situações de evidências clínicas incertas, onde há riscos à saúde, sendo as preferências individuais determinantes para a decisão.

O rastreamento do câncer de próstata é uma dessas situações de incerteza, onde as evidências científicas atuais questionam os benefícios de se realizar o exame de Antígeno Prostático Específico (PSA) e o toque retal como rotina, que podem ocasionar danos subsequentes aos procedimentos e ao tratamento de cânceres de baixa letalidade..$^{8,9}$ As complicações decorrentes mais comuns são: dor, sangramento e infecção pela biópsia; problemas no intestino, incontinência urinária e impotência sexual pelo tratamento que afetam a qualidade de vida do homens. ${ }^{8}$

Por tais incertezas, diversas instituições nacionais e internacionais, assim como o Ministério de Saúde do Brasil, não recomendam a realização de programas de rastreamento populacional para o câncer de próstata. Homens assintomáticos que demandarem esses exames devem ter acesso à informação sobre as possíveis implicações e decidir, de forma conjunta com o médico, se desejam ou não realizá-los. ${ }^{10}$

No entanto, mesmo diante dessa recomendação, dados do Sistema de Informação Ambulatorial no Brasil (SIA/SUS) demonstram aumento progressivo do número exames de PSA realizados ao longo dos anos. Embora não seja possível distinguir na base de dados a finalidade do exame (rastreamento ou diagnóstico), esse aumento sugere a prática do rastreamento e o pressuposto de que há dificuldades na comunicação dos riscos e benefícios na efetivação da decisão compartilhada para tal escolha. ${ }^{11}$

Como o rastreamento está voltado para indivíduos saudáveis, há maior preocupação ética com essa prática, pois, os riscos de danos não estão contrabalançados com um sofrimento real, mas sim, ancorados em uma possibilidade futura de adoecimento e morte. Nesse caso, o princípio de não causar danos deve imperar, visto que pessoas assintomáticas, que se percebem como saudáveis, podem ter sua saúde abalada devido à intervenção. ${ }^{12}$ Essa perspectiva dialoga com a Prevenção Quaternária, que tem por intuito proteger os indivíduos dos malefícios ocasionados por excessivas intervenções médicas ${ }^{13-15}$.

Apesar de referenciais que valorizem a decisão clínica compartilhada para o cuidado integral, partiuse do pressuposto que ainda há dificuldades de implementá-los nas práticas da APS, especialmente no rastreamento do câncer de próstata. Para explorar tais contradições, o objetivo desse estudo foi analisar a percepção e experiências de homens e médicos da APS/SUS sobre a tomada de decisão compartilhada para o rastreamento do câncer de próstata.

\section{MÉTODO}

Trata-se de um estudo qualitativo, exploratório, com dados primários que compõem uma primeira etapa de um projeto para o desenvolvimento de uma ferramenta de decisão clínica para o rastreamento do câncer de próstata. A ferramenta foi disponibilizada pelo Instituto Nacional do Câncer, em 2020, tendo como subsídios, as análises inéditas deste artigo. ${ }^{16}$

Como técnica de coleta, foi realizado um grupo focal com homens que trabalhavam em uma empresa de energia no Rio de Janeiro ( $n=19)$, no ano de 2019. Procurou-se como elegibilidade, selecionar homens 
adultos, na maturidade etária (em torno de 40 anos), saudáveis, em ambiente comunitário. Justifica-se a escolha desse local pelo fato de a gestão da empresa possuir um programa de educação em saúde e ter estabelecido parcerias institucionais sobre o tema prevenção de câncer. Realizou-se convite aberto aos trabalhadores para participação, de forma voluntária, em encontro da pesquisa, ocasionando o comparecimento de um número até mesmo superior ao previsto. ${ }^{17}$

O grupo foi realizado durante um turno de trabalho, com a presença de dois facilitadores experientes na técnica, para mediar a participação de todos, sem a presença de outras pessoas da empresa. Foi utilizado um roteiro com cinco perguntas norteadoras, que possuíam o intuito de provocar discussão sobre a percepção dos homens em relação aos exames de rastreamento do câncer de próstata, os motivos para sua realização, conhecimento sobre os seus possíveis riscos e benefícios e, por fim, obter relatos de experiências de participação (ou ausência) na tomada de decisão. O perfil dos homens foi coletado por um questionário de preenchimento individual, com auxílio dos facilitadores, no início do grupo.

Realizou-se ainda entrevista semiestruturada com médicos que atuavam na APS em diferentes regiões do país no ano de 2019, considerando as atribuições deste nível de atenção na detecção precoce do câncer. ${ }^{10,11}$ Os médicos elegíveis receberam o convite por e-mail, telefone e/ou aplicativos de mensagens, após os pesquisadores solicitarem divulgação do estudo para as suas redes de contato, sendo estes, pesquisadores, preceptores de residência de MFC, gestores e outros profissionais da APS. O convite explicitava os objetivos da pesquisa, os critérios para participação e solicitava que os interessados entrassem em contato com a pesquisadora principal por telefone, mensagem ou e-mail. Ademais, foi solicitado que os médicos entrevistados indicassem novos médicos para serem convidados.

Os pesquisadores enviaram 93 convites, incluindo contatos prévios ou indicação de participantes, que gerou 42 respostas de médicos interessados em participar da pesquisa, porém, seis informaram não conseguirem concluir todas as etapas do estudo e 21 não retornaram o contato para o agendamento da entrevista, mesmo após três tentativas. Ao final, 15 médicos de 11 estados brasileiros participaram das entrevistas remotas (duração entre 30-40 minutos/cada).

As entrevistas foram guiadas por um roteiro de 11 perguntas, previamente testado e modificado (após aplicação em dois médicos e um especialista em detecção precoce do câncer), contemplando os seguintes temas: a prática médica no rastreamento do câncer próstata, a percepção sobre a decisão compartilhada, as barreiras e facilidades para sua implementação; o conhecimento e comunicação sobre os riscos e benefícios dos exames de rastreamento e, por último, a utilização de ferramenta de apoio à decisão. O perfil do médico e da área da atuação foram capturados no início das entrevistas.

Os instrumentos de pesquisa foram produzidos pelos pesquisadores e todo material coletado foi gravado, transcrito na íntegra, submetido a pré-análise (leitura exaustiva, sistematização e categorização) e analisado, a partir de duas categorias temáticas: Proteção da saúde X Riscos do rastreamento e Valorização da autonomia dos homens na tomada de decisão clínica ${ }^{17}$.

Esse estudo está em consonância com as diretrizes apresentadas pelo referencial metodológico para pesquisa qualitativa (Coreq) e atendeu aos preceitos éticos das pesquisas em seres humanos, sendo aprovado pelo CEP/ENSP em 2015, CAAE12165019.4.0000.5240. 


\section{RESULTADOS}

Os homens participantes do grupo possuíam idade variando de 38 a 72 anos (média= 51 anos) e variados níveis de escolaridade e função laboral. Os médicos/as entrevistados tinham idade de 25 a 46 anos (média=35 anos) e mais da metade era do sexo feminino, o que de certa forma reflete a feminização das profissões de saúde e da demografia médica no país ${ }^{18}$ e nos remete a refletir acerca de eventuais dificuldades de gênero para abordar a saúde do homem (tabela 1).

Destaca-se também que os médicos/as possuíam pouco tempo de formação (< 5 anos) e um pouco mais da metade possuía residência em medicina de família e comunidade, experiência e formação que poderiam contribuir para decisões clínicas compartilhadas. Quanto ao local de atuação, os médicos/as eram dos seguintes estados: Espírito Santo, Minas Gerais (2), Rio de Janeiro (2), Paraná, Paraíba (2), São Paulo, Santa Catarina, Rio grande do Sul, Goiás, Rondônia, Sergipe (2), variando de áreas urbana $(60 \%)$ e rural $(40 \%)$ (tabela 2$)$.

Considerando a existência dialógica de modelos biomédicos/intervencionistas, em contraposição às práticas inclusivas ancoradas na decisão compartilhada, a seguir, apresenta-se as categorias analíticas, com os sentidos e experiências dos participantes, homens e médicos/as, de forma integrada.

\section{Proteção da saúde $X$ Riscos do rastreamento}

Há convergência na percepção de homens e médicos/as sobre a importância de medidas de prevenção para o câncer de próstata; mas em contrapartida, nota-se certo confundimento sobre fazer o rastreamento como forma de evitar a doença, uma vez que, os motivos para sua realização parecem estar pautados na proteção da saúde, desconsiderando, na maioria das vezes, os riscos envolvidos.

Tabela1. Perfil do grupo de homens.

\begin{tabular}{llc}
\hline & N & $\%$ \\
\hline Grupo de homens & $\mathbf{1 9}$ & \\
\hline Idade & & $0 \%$ \\
\hline 25 a 29 anos & 0 & $21 \%$ \\
30 a 39 anos & 4 & $16 \%$ \\
40 a 49 anos & 3 & $37 \%$ \\
50 a 59 anos & 7 & $26 \%$ \\
$\geq 60$ anos & 5 & \\
\hline Estado civil & & $63 \%$ \\
\hline Casado & 12 & $32 \%$ \\
Solteiro & 6 & $5 \%$ \\
Viúvo & 1 & \\
\hline Nível de escolaridade & & \\
\hline Fundamental & 4 & $32 \%$ \\
Médio & 6 & $5 \%$ \\
Superior incompleto & 1 & \\
Superior completo & 8 & \\
\hline
\end{tabular}


Tabela 2. Perfil dos médicos/as.

\begin{tabular}{|c|c|c|}
\hline Médicos/as & 15 & \\
\hline \multicolumn{3}{|l|}{ Idade } \\
\hline 25 a 29 anos & 8 & $53 \%$ \\
\hline 30 a 39 anos & 4 & $27 \%$ \\
\hline 40 a 49 anos & 2 & $13 \%$ \\
\hline 50 a 59 anos & 0 & $0 \%$ \\
\hline$\geq 60$ anos & 1 & $7 \%$ \\
\hline \multicolumn{3}{|l|}{ Gênero } \\
\hline Feminino & 9 & $60 \%$ \\
\hline Masculino & 6 & $40 \%$ \\
\hline \multicolumn{3}{|l|}{ Tempo de graduação } \\
\hline $1 \mathrm{e}<2$ anos & 6 & $40 \%$ \\
\hline$\geq 2 \mathrm{e}<5$ anos & 4 & $27 \%$ \\
\hline$\geq 5 \mathrm{e}<8$ anos & 4 & $27 \%$ \\
\hline$\geq 8$ anos & 1 & $7 \%$ \\
\hline \multicolumn{3}{|l|}{ Especialização } \\
\hline Residência em Medicina de Família e Comunidade & 8 & $53 \%$ \\
\hline Outra & 1 & $7 \%$ \\
\hline Não possui & 6 & $40 \%$ \\
\hline \multicolumn{3}{|l|}{ Região } \\
\hline Norte & 1 & $7 \%$ \\
\hline Nordeste & 4 & $27 \%$ \\
\hline Sudeste & 6 & $40 \%$ \\
\hline Centro-Oeste & 1 & $7 \%$ \\
\hline Sul & 3 & $19 \%$ \\
\hline \multicolumn{3}{|l|}{ Área de atuação } \\
\hline Urbana & 9 & $60 \%$ \\
\hline Rural & 6 & $40 \%$ \\
\hline
\end{tabular}

O interesse dos homens pelos exames ficou evidente, todos informaram acreditar que deveriam ser realizados de forma rotineira, exceto um que preferiu não opinar; dentre esses, todos informaram desconhecer qualquer probabilidade de risco. Mais da metade dos homens (60\%) informou já ter realizado algum dos exames, com a justificativa de preservar a saúde e prevenir o adoecimento e morte por câncer de próstata. Os médicos referem receber constante solicitação dos seus usuários, principalmente durante campanhas midiáticas.

"Faço para manter-me saudável e sem risco para minha saúde, pois entendo que é necessário para poder prevenir o câncer de próstata e preservar a minha vida." H19

“...quando a gente fala com o paciente, ele não tem noção sobre os riscos, ele tá focado no medo, de ter o câncer e não pensa nessa questão do exame que pode dar falsos positivos e quantos evoluem para fazer cirurgia sem precisar." M11 
As narrativas dos médicos/as também mencionam dificuldades para discutir os riscos do rastreamento. Alguns relatam que essas informações são recebidas com desconfiança e, às vezes, atreladas à necessidade de poupar recursos financeiros pelos serviços.

“Se eu não pedir, o paciente fala que vai a um médico particular pegar o pedido"M14

"Quando ele pede, eu paro, falo que tem todos esses problemas, esses riscos aí, alguns aceitam, entendem... mas é um processo difícil, alguns não ficam satisfeitos M5.

A prática da solicitação do exame foi contraditória nos relatos dos médicos/as. A maioria (60\%) referiu não indicar o rastreamento, justamente pelos riscos envolvidos e por seguir a recomendação de órgão oficiais, no entanto, alguns (40\%), mesmo conhecendo os riscos, afirmaram estimular a realização, justificando acreditar na efetividade para diagnosticar precocemente o câncer, e assim, proporcionar os benefícios do tratamento em estágios iniciais.

"Quando o homem vem na consulta, pede pelo check-up, que é o PSA... Eu já explico que agora não é mais recomendado fazer, tem os problemas do risco... Também tem a sociedade [Sociedade Brasileira de Medicina de Família e Comunidade] que já diz que não é para fazer, então explico que é melhor não fazer." M2

"A gente aprende que, a partir de uma certa idade, é interessante que todo homem faça, mesmo não sendo correto pelo Ministério da Saúde, mas para tirar dúvida a gente sempre passa e oferece pros pacientes." M14

A despeito das contradições entre as medidas de prevenção, rastreamento e diagnóstico precoce, ficou evidenciado que a realização do exame se dá, essencialmente, por medo de uma doença que possui uma elevada carga de dor, incapacidade e morte.

"Muitos pedem o exame porque tem medo de acontecer algo mais para frente, o medo de ter um diagnóstico, medo do que aconteceria com eles." M10

O câncer ainda desencadeia um forte sentimento nos profissionais e na população de que "vale tudo para combater essa doença". Esse sentimento fortalece as atitudes de que o rastreamento, seguido de tratamento precoce, é a abordagem mais adequada para o câncer. ${ }^{19}$ No entanto, evidências atuais demonstram que o câncer de próstata pode ter evolução indeterminada, e assim, o rastreio pode identificar mais indivíduos doentes que o necessário e provocar malefícios à saúde. ${ }^{8}$

Nos relatos dos médicos/as é possível perceber que há um receio particular de não solicitar esses exames, que pode estar atrelado à concepção de que a prevenção e a proteção à saúde necessariamente demandam intervenções médicas, já que reconhecem a contraindicação do rastreamento. Esse é um modo 
de atuação comum na clínica e pode ter como consequência a redução da autonomia e da competência das pessoas para manejar adversidades na sua saúde, gerando demasiada dependência da ação do profissional. ${ }^{15}$

Essas medidas intervencionistas somam-se às práticas da medicina defensiva, que resulta no emprego de condutas excessivas, com o objetivo de evitar a judicialização e outras complicações legais relativas aos atos médicos. ${ }^{20}$ Uma pesquisa realizada no estado de São Paulo identificou que, $75 \%$ dos médicos respondentes afirmaram que o medo de ser acionado judicialmente interfere na sua prática e mais de $90 \%$ concordaram com a afirmativa de que solicitam mais exames que o necessário, como forma de se proteger contra processos. ${ }^{21} \mathrm{~A}$ literatura aponta que a medicina defensiva pode gerar a utilização desmedida de tecnologias diagnósticas, de forma a minimizar o sentimento de insegurança dos profissionais. ${ }^{20,22}$

Segundo os preceitos da Prevenção Quaternária, o uso racional dos recursos de saúde promove o emprego consciente dos dispositivos tecnológicos, diminui a probabilidade de adoecimento iatrogênico, valoriza a ética do cuidado e estimula o pensamento crítico dos profissionais para avaliar e proteger as pessoas de danos induzidos pelas intervenções médicas. ${ }^{13,14}$

Apoiados nessa premissa e acrescidos da ética do cuidado, movimentos médicos buscam difundir essas informações, como um exemplo, o choosing wisely, que dentre as principais recomendações orientam a não realização do rastreamento do câncer de próstata e apresenta o estímulo midiático, a pressão dos pacientes por mais exames, pagamento por serviços, além do marketing da indústria farmacêutica e de equipamentos, como os principais catalizadores do consumo de procedimentos. ${ }^{13,14,23}$

Devido à magnitude do câncer de próstata, há preocupação dos médicos/as e população sobre as possíveis medidas para seu controle. Porém, evidências atuais demonstram o limite do rastreamento como resposta a esse problema, diferentemente das ações de diagnóstico precoce, por meio dos sinais e sintomas, seguido de acesso oportuno e adequado ao tratamento. ${ }^{10} \mathrm{O}$ confundimento entre essas práticas e seus benefícios ficou evidenciado quando elencados os motivos para a solicitação/indicação do rastreamento. Os homens acreditam estar protegendo sua saúde frente a uma doença que causa medo; os médicos/as enfrentam o imperativo social quanto a sua realização, o receio da responsabilização por negligência e também suas próprias crenças.

\section{Valorização da autonomia dos homens na tomada de decisão clínica}

Os resultados demostraram que os homens referem desejo de maior participação na decisão, justificando acreditar ser imprescindível sua opinião diante de uma decisão que afeta diretamente sua qualidade de vida e bem-estar. Os médicos/as reconheceram a importância de incluir os homens na decisão, no entanto, identificam fatores que dificultam essa abordagem.

"Deveria sempre acontecer [incluir o homem na decisão], pois são coisas que acontecerão com o meu corpo e que irá afetar o meu modo de vida e da minha família"H6. 
“Eu acho importante conversar com o paciente, falar que a decisão no fundo é dele... abrir um pouco o horizonte do paciente, que não é só o exame, mas é uma cadeia e que há complicações importantes para vida dele... é importante que ele saiba, e que esteja ciente do seu cuidado." M13

Os médicos/as que referiram incluir o homem na decisão (60\%) relatam maior satisfação dos usuários, porém, ressaltam que é comum encontrar comportamentos que reproduzem uma lógica paternalista de cuidado, em que o médico/a é visto como o único responsável pela decisão.

“... Eu vejo que alguns gostam, ficam felizes, mas outros estranham... tem uma visão em relação ao médico que é o que determina tudo, e quando falo das opções, ele rebate, "você é o médico"”. M3

"O médico é você, o que falar, está decidido", e aí tento contornar, dizendo que hoje em dia é uma forma diferente de abordar, é o paciente presente, dividindo, porque é ele que vai ser exposto a esse tipo de procedimento... eles rebatem muito no sentido de, "quem decide é vc" entrega toda a responsabilidade para gente, por estar ali meramente passivo. Alguns eu consigo dividir, eles entendem, mas a primeira resposta é "você decide"'". M15

A vontade de estar envolvido na decisão pode variar de acordo com a situação clínica e características individuais ${ }^{24}$, mas entende-se que essa interação deve ser estabelecida após diálogo aberto, considerando a vontade individual. ${ }^{1} \mathrm{O}$ vínculo também pode influenciar a construção dessa relação, seja pelo seu componente temporal, expresso pela longitudinalidade do cuidado, e/ou o interpessoal, por meio da empatia e acolhimento. ${ }^{25}$

Ademais as dificuldades de comunicação, os médicos/as também identificaram a baixa escolaridade e o nível socioeconômico como barreiras para estabelecer a decisão compartilhada.

“Os meus pacientes são bem simples, então eu já falo logo o que é melhor para eles”.M9

"Meus pacientes são idosos, bem fáceis de lidar, se você fala que é isso, é isso, não discutem, não entendem muito, na verdade eles nem são tão curiosos." M1

Esse argumento resulta da concepção de que assumir um papel ativo no processo de tomada de decisão é particularmente difícil para populações vulneráveis ${ }^{1}$, o que demonstra preconceito em relação à capacidade de entendimento e interação dessas pessoas. Essa distinção pode ocasionar disparidades em saúde, já que a participação no cuidado está relacionada a melhores resultados clínicos. ${ }^{26}$ Estudos internacionais apresentam esforços para diminuir disparidades na comunicação clínica, como o desenvolvimento de ferramentas que buscam adequar a linguagem e a forma de transmitir a informação. ${ }^{27,28}$ Esse movimento foi percebido na fala de alguns médicos/as entrevistados, que relataram utilizar recursos para incluir diferentes perfis de homens na tomada de decisão. 
"Eu às vezes uso figurinhas, tipo pictogramas da internet para mostrar os pacientes esses riscos, mostrando os desenhos, eles visualizam e entendem mais.... O bom é que não fica só eu falando. M3"

"Geralmente eu falo muito sobre os riscos, mas às vezes parece que eles não estão entendendo, daí eu repito e peço para me dizer o que entendeu..." M10

Dentre os fatores que influenciam a decisão compartilhada, a literatura internacional aponta a postura do profissional como um importante facilitador, a forma de abordagem durante a consulta pode influenciar na capacidade e vontade do usuário de participar. Demais fatores referem-se ao estilo de comunicação, que deve ser clara e facilmente compreensível, além da necessidade de dispensar tempo para esclarecer dúvidas. ${ }^{1,29}$

A utilização da internet também foi identificada com um fator que influencia a relação médico-paciente. Os homens relataram recorrer a esse recurso com o intuito de adquirir informações e os médicos/as da mesma forma, referem receber, de forma frequente, pedidos dos homens para realizar o rastreamento do câncer de próstata, referindo ter lido sobre sua importância em redes sociais virtuais ou aplicativos de mensagens.

“....hoje em dia se o médico não falar, a gente busca na internet e fica sabendo de tudo...” H12

“... o paciente vai até a internet e pesquisa no Google e já chega com toda a informação”. M12.

A crescente busca sobre saúde na internet pode proporcionar benefícios relacionados ao aumento do conhecimento, porém, é necessário atentar para as fontes da informação. ${ }^{30}$ Um estudo demonstrou que dentre os vídeos disponíveis sobre câncer de próstata em uma plataforma digital, $77 \%$ foram considerados potencialmente desinformativos e $27 \%$ foram classificados como tendenciosos comercialmente. Os vídeos considerados de mais alta qualidade tiveram o menor número de visualizações por mês e os que possuíam conteúdo questionável foram os mais acessados. ${ }^{31,32}$

Outra barreira identificada pelos médicos/as foi a organização do processo de trabalho voltado para a lógica produtivista. $O$ excesso de trabalho e a falta de tempo apareceram como fatores que impossibilitam dispensar mais tempo nas tecnologias relacionais.

“Nem sempre dá tempo de explicar tudo para o paciente, pois enquanto você está falando já tem outro na porta esperando".M6

Na medida em que a rotina do trabalho sufoca o espaço de partilha, há prejuízo da relação interpessoal, que leva à negligência de um dos princípios éticos fundamentais do cuidado - a autonomia. ${ }^{33,34} \mathrm{~A}$ autonomia está associada à consolidação de direitos humanos e no contexto da relação médico-paciente está associada ao respeito e reconhecimento da pessoa como capaz de influenciar o seu cuidado e, assim de forma compartilhada, alcançar a opção mais adequada ao seu contexto de vida. ${ }^{35}$ 
Estudos internacionais apresentam resultados semelhantes a esse estudo no que se refere à vontade dos pacientes de serem incluídos na decisão, no entanto, apresentam barreiras individuais e estruturais do sistema de saúde. ${ }^{36-38}$

\section{Implicações para a prática profissional}

Os resultados demonstram como os profissionais e homens percebem e experienciam o compartilhamento da decisão sobre o rastreamento câncer de próstata, demostrando exemplos de práticas inclusivas, embora ainda revele ações médico-centradas e intervencionistas. Os achados também apresentam estratégias utilizadas pelos médicos, como os recursos gráficos e ferramentas de comunicação clínica, que ajudam a incluir diferentes perfis de homens na tomada de decisão.

Nessa perspectiva, projetamos gerar discussão na maneira de realizar a clínica, principalmente diante de intervenções que possuem evidências incertas, como o rastreamento do câncer de próstata, mesmo que signifique ocasionar ruptura na prática rotineira do profissional. Além disso, cabe ampliar o debate sobre as implicações do rastreamento entre os profissionais inseridos na APS e na população.

Dessa forma, fazem-se necessárias estratégias educacionais, seja na formação ou educação permanente, para o desenvolvimento de habilidades relacionais, buscando valorizar a autonomia e a participação individual na decisão, uma vez que, identificou-se vontade dos homens de conhecerem as implicações das intervenções biomédicas em sua saúde. Da mesma forma, sugere-se o diálogo aberto e transparente durante a relação clínica, de modo a incentivar a participação dos homens na decisão, já que muitos não se reconhecem como parte desse processo.

De forma a facilitar essa relação, aconselha-se a utilização de estratégias e ferramentas de comunicação clínica que possam auxiliar na discussão e entendimento sobre os riscos e benefícios do rastreamento do câncer de próstata. Uma ferramenta de acesso gratuito publicada pelo INCA/MS, se encontra disponível em:

https://www.inca.gov.br/sites/ufu.sti.inca.local/files//media/document/apoio decisao cancer prostata 2019 0.pdf.

\section{Considerações finais}

A investigação sobre a percepção e a experiência de homens e médicos sobre compartilhar a decisão para o rastreamento do câncer de próstata demonstrou que, embora seja uma recomendação de órgãos oficiais, no Brasil, essa prática ainda não está consolidada, apesar de avanços em relação ao debate de possíveis implicações danosas e no reconhecimento da importância de incluir o homem na tomada de decisão.

A maioria dos médicos/as relatam empenho para deliberar sobre as consequências do rastreamento, no entanto, enfrentam dificuldades estruturais, que se relacionam com questões culturais, pessoais e pouco domínio sobre a organização do processo de trabalho. Essas barreiras dificultam o processo partilha e impossibilitam dispensar maior tempo nas tecnologias relacionais durante a consulta. 
Esforços para implementar a decisão compartilhada se justificam a partir do desejo dos homens em participar mais ativamente nas escolhas sobre o seu cuidado e devido à iminente necessidade de romper com um modelo paternalista de cuidado, por vezes, reproduzido pelos próprios usuários.

Como limites do estudo, destaca-se a seleção por conveniência dos participantes, que pode ter contribuído para a inclusão de indivíduos mais sensíveis e interessados no tema. Em contrapartida, destacase a inserção dos médicos/as entrevistados de diferentes estados do Brasil, permitindo ter uma visão de diferentes cenários da APS. Além disso, foi possível explorar a percepção dos médicos/as e homens, principais atores, sobre os fatores que estimulam e influenciam a prática do rastreamento do câncer de próstata e como a participação individual e a autonomia estão implicadas na relação clínica.

Outra contribuição do estudo foi a possibilidade de identificar de forma qualitativa as dificuldades que esses atores enfrentam diante de um tema com intensa divergência e muito difundido pelos meios de comunicação, que muitas vezes disseminam informações que prejudicam o cuidado.

Perspectivas futuras sobre o tema referem-se à importância de investir em estratégias e recursos que facilitem a comunicação médico/paciente, buscando consolidar a coparticipação dos usuários/as nas decisões sobre sua saúde. E para além da temática do câncer de próstata, há a necessidade de ampliar o debate sobre os riscos/benefícios das intervenções em saúde, com intuito de promover a deliberação sobre outras ações de rastreamento, comuns na prática clínica.

Por fim, destaca-se que história, relevância e tendência geral da atualidade demonstram que a decisão compartilhada não é apenas uma proposta do momento, mas sim uma abordagem necessária que irá desempenhar um papel cada vez mais importante na maneira como pensamos nossa saúde e nossa relação com o cuidado, o que demanda o importante exercício de se valorizar a relação interpessoal nos cenários clínicos.

\section{Conflitos de interesse}

Os autores declaram não haver conflito de interesse.

\section{Declaração autoria}

Renata Oliveira Maciel dos Santos. Concepção do estudo, realização da coleta de dados, análise dos dados, discussão dos achados, redigiu o manuscrito.

Mirhelen Mendes de Abreu. Revisão dos dados, discussão dos achados e aprovação da versão final.

Elyne Montenegro Engstrom. Concepção do estudo, revisão dos dados, discussão dos achados e aprovação da versão final.

\section{REFERÊNCIAS BIBLIOGRÁFICAS}

1. Hargraves IG, Montori VM, Brito JP, Kunneman M, Shaw K, LaVecchia C, et al. Purposeful SDM: A problem-based approach to caring for patients with shared decision making. Patient Education and Counseling.2019;102(10):1786-92. https://doi.org/10.1016/j.pec.2019.07.020

2. Elwyn G, Durand MA, Song J, Aarts J, Barr PJ, Berger Z, et al. A three-talk model for shared decision making: multistage consultation process. BMJ. 2017;359. https:// doi: 10.1136/bmj.j4891

3. Stewart M, Brown J, weston W, Mcwhiney I, Mcwillian C, Freeman T. Medicina centrada na pessoa: transformando o método clínico. 30 ed. Artmed; 2017. 
4. Brasil. Ministério da Saúde. Política Nacional de Humanização: PNH [Internet]. Brasília: Ministério da Saúde;2013 [acesso 10 Nov 2020$].$ Disponível: https://bit.ly/2X37vvl

5. Brasil. Ministério da Saúde. Política Nacional de Promoção da Saúde: PNPS [Internet]. Brasília: Ministério da Saúde; 2017 [acesso 15 Nov 2020]. Disponível em: http://bvsms.saude.gov.br/bvs/saudelegis/gm/2014/prt2446_11_11_2014.html

6. Brasil. Portaria de consolidação. Política Nacional de Atenção Básica. Artigo 6o Anexo XXII [Internet]. Ministério da Saúde; 2017. Disponível em: http://bvsms.saude.gov.br/bvs/saudelegis/gm/2017/prc0002_03_10_2017.html

7. Abreu MM de, Battisti R, Martins RS, Baumgratz TD, Cuziol M. Shared decision making in Brazil: history and current discussion. Zeitschrift für Evidenz, Fortbildung und Qualität im Gesundheitswesen [Internet]. 2011;105(4):240-4. DOI: 10.1016/j.zefq.2011.04.009

8. Fenton JJ, Weyrich MS, Durbin S, Liu Y, Bang H, Melnikow J. Prostate-Specific Antigen-Based Screening for Prostate Cancer: Evidence Report and Systematic Review for the US Preventive Services Task Force. JAMA [Internet]. 2018;319(18):1914-31. DOI: 10.1001/ jama.2018.3712

9. Santos ROM, Ramos DN, de Assis M. Construção compartilhada de material educativo sobre câncer de próstata. Revista Panamericana de Salud Pública [Internet]. 2018; 42:1-8. https://doi.org/10.26633/RPSP.2018.122

10. Instituto Nacional de Câncer José Alencar Gomes da Silva. Diretrizes para a detecção precoce do câncer de mama no Brasil [Internet]. Rio de Janeiro: Inca; 2015 [acesso 18 Ago 2020]. Disponível: https://bit.ly/3bPqKMP

11. Instituto Nacional de Câncer José Alencar Gomes da Silva. Monitoramento das ações de controle do câncer de próstata. Informativo detecção precoce; 2017. Boletim ano 8, n.2, julho/ dezembro [acesso 11 Jan 2020]. Disponível: https://bit.ly/3bPqKMP

12. Norman AH. Medical ethics and screening: on what evidence should we support ourselves? Revista Brasileira de Medicina de Família e Comunidade 2014 [citado 18 de abril de 2020];9(31):108-10. http://dx.doi.org/10.5712/rbmfc9(31)933

13. Norman AH, Tesser CD. Prevenção quaternária: as bases para sua operacionalização na relação médico-paciente. Revista Brasileira de Medicina de Família e Comunidade. 2015; 10(35): 1-10, abr.-jun. https://doi.org/10.5712/rbmfc10(35)1011

14. Norman AH, Tesser CD. Quaternary prevention: a balanced approach to demedicalisation. - [Internet]. British Journal of General Practice. 2019; 69 (678): 28-29. https://doi.org/10.3399/bjgp19X700517

15. Tesser CD. Convergências entre prevenção quaternária e promoção da saúde. Revista Brasileira de Medicina de Família e Comunidade. 2020;15(42):2515-2515. DOI: https://doi.org/10.5712/rbmfc15(42)2515

16. Santos ROM, Engstrom EM, Abreu MM. Minha vida, minha voz na decisão: decisão compartilhada no rastreamento do câncer de próstata. Tese de doutorado. [Rio de Janeiro, RJ]: Fundação Oswaldo Cruz, Escola Nacional de Saúde Pública Sergio Arouca. 2020.

17. Uwe Flick. Introdução à pesquisa qualitativa. 3a. Porto Alegre: Artmed; 2009.

18. SCHEFFER, M. et al. Demografia médica no Brasil 2018. São Paulo, SP: FMUSP, CFM, Cremesp; 2018.

19. Aronowitz R, Greene J. Contingent Knowledge and Looping Effects - A 66-Year-Old Man with PSA-Detected Prostate Cancer and Regrets. N Engl J Med. 2019; 381:1093-1096. https://doi.org/ 10.1056/NEJMp1811521

20. Minossi JG, Silva AL da. Medicina defensiva: uma prática necessária? Rev. Col. Bras. Cir. [online]. 2013, vol.40, n.6, pp.494-501. https:// doi.org/10.1590/S0100-

21. Vale HM do, Miyazaki MC de OS, Vale HM do, Miyazaki MC de OS. Medicina defensiva: uma prática em defesa de quem? Revista Bioética. 2019;27(4):747-55. http://doi.org/10.1590/1983-80422019274358

22. Vasconcelos C. Responsabilidade médica e judicialização na relação médico-paciente. Revista Bioética. [internet]. 2012;20(3) [acesso 11 Jan 2020]. Disponível em: http://revistabioetica.cfm.org.br/index.php/revista_bioetica/article/view/757

23. Laguardia J, Martins MS, Barcellos GB. Qualidade do cuidado em saúde e a iniciativa “Choosing Wisely”. RECIIS (Online). 2016; 10(1):18. https://doi.org/10.29397/reciis.v10i1.1097

24. Silva CID. Quando o antígeno específico da próstata nos leva ao Balint: um relato de caso. Revista Brasileira de Medicina de Família e Comunidade. 2017;12(39):1-6. https://doi.org/10.5712/rbmfc12(39)1518

25. Santos ROM dos, Romano VF, Engstrom EM. Vínculo longitudinal na Saúde da Família: construção fundamentada no modelo de atenção, práticas interpessoais e organização dos serviços. Physis [online]. 2018, vol.28, n.2. http://dx.doi.org/10.1590/s0103-73312018280206.

26. Légaré F, Thompson-Leduc P.Twelve myths about shared decision making. Patient Education and Counseling.2014;96(3):281-6. https:// doi.org/10.1016/j.pec.2014.06.014 
27. Scalia P, Durand M-A, Faber M, Kremer JA, Song J, Elwyn G. User-testing an interactive option grid decision aid for prostate cancer screening: lessons to improve usability. BMJ Open. 2019;9(5): https://doi.org/ 10.1136/bmjopen-2018-026748

28. Stacey D, Légaré F, Lewis K, Barry MJ, Bennett CL, Eden KB, et al. Decision aids for people facing health treatment or screening decisions. Cochrane Database of Systematic Reviews. 2017. https://doi.org/10.1002/14651858.CD001431.pub5/full

29. Steffensen KD, Vinter M, Crüger D, Dankl K, Coulter A, Stuart B, et al. Lessons in Integrating Shared Decision-Making Into Cancer Care. J Oncol Pract. 2018;14(4):229-35. https://doi.org/10.1200/JOP.18.00019

30. Iverson SA, Howard KB, Penney BK. Impact of Internet Use on Health-Related Behaviors and the Patient-Physician Relationship: A Survey-Based Study and Review. J Am Osteopath Assoc 2008;108(12):699-711. [acesso em 11 Jan 2020]. Disponível em: https:// europepmc.org/article/med/19075034

31. Carneiro B, Dizon DS. Prostate Cancer Social Media: In YouTube We Trust? Eur Urol. 2019;75(4):568-9. https://doi.org/10.1016/j. eururo.2019.01.004

32. Loeb S, Sengupta S, Butaney M, Macaluso JN, Czarniecki SW, Robbins R, et al. Dissemination of Misinformative and Biased Information about Prostate Cancer on YouTube. European Urology. 2019;75(4):564-7. https://doi.org/10.1016/j.eururo.2018.10.056

33. Nunes FDBRS, Almeida A das DL de. Informação médica e consentimento de pessoas com câncer. Revista Bioética. 2018;26(1):119-26. https://doi.org/10.1590/1983-80422018261233

34. Pieterse AH, Stiggelbout AM, Montori VM. Shared Decision Making and the Importance of Time. JAMA [Internet]. 2019;322(1):25-6. https://doi.org/10.1001/jama.2019.3785

35. Marques Filho J, Hossne WS. A relação médico-paciente sob a influência do referencial bioético da autonomia. Revista Bioética. 2015;23(2):304-10. https://doi.org/10.1590/1983-80422015232069

36. Hahlweg P, Witzel I, Müller V, Elwyn G, Durand M-A, Scholl I. Adaptation and qualitative evaluation of encounter decision aids in breast cancer care. Arch Gynecol Obstet. 2019;299(4):1141-9. https://doi.org/10.1007/s00404-018-5035-7

37. Menear M, Garvelink MM, Adekpedjou R, Perez MMB, Robitaille H, Turcotte S, et al. Factors associated with shared decision making among primary care physicians: Findings from a multicentre cross-sectional study. Health Expect. 2018;21(1):212-21. https://doi. org/10.1111/hex.12603

38. Hajizadeh N, Uhler LM, Pérez Figueroa RE. Understanding patients' and doctors' attitudes about shared decision making for advance care planning. Health Expect. dezembro de 2015;18(6):2054-65. https://doi.org/10.1111/hex.12285 